\title{
Fiber-Centered Analysis of Brain Connectivities Using DTI and Resting State FMRI Data
}

\author{
Jinglei $\mathrm{Lv}^{1}$, Lei Guo ${ }^{1}$, Xintao $\mathrm{Hu}^{1}$, Tuo Zhang ${ }^{1}$, Kaiming $\mathrm{Li}^{1,2}$, Degang Zhang ${ }^{1,2}$, \\ Jianfei Yang ${ }^{1}$, and Tianming Liu $^{2}$ \\ ${ }^{1}$ School of Automation, Northwestern Polytechnical University, Xi' an, China \\ ${ }^{2}$ Department of Computer Science and Bioimaging Research Center, \\ The University of Georgia, Athens, GA, USA
}

\begin{abstract}
Recently, inference of functional connectivity between brain regions using resting state fMRI (rsfMRI) data has attracted significant interests in the neuroscience community. This paper proposes a novel fiber-centered approach to study the functional connectivity between brain regions using high spatial resolution diffusion tensor imaging (DTI) and rsfMRI data. We measure the functional coherence of a fiber as the time series' correlation of two gray matter voxels that this fiber connects. The functional connectivity strength between two brain regions is defined as the average functional coherence of fibers connecting them. Our results demonstrate that: 1) The functional coherence of fibers is correlated with the brain regions they connect; 2) The functional connectivity between brain regions is correlated with structural connectivity. And these two patterns are consistent across subjects. These results may provide new insights into the brain's structural and functional architecture.
\end{abstract}

Keywords: functional network, structural network, rsfMRI, DTI, functional coherence.

\section{Introduction}

Recently analysis of functional connectivity between brain regions using resting state fMRI (rsfMRI) data has attracted significant interests in neuroscience community [13]. Functional connectivity between two brain regions is typically defined as the similarity of their fMRI time series. One of the major challenges in functional connectivity analysis of the human brain is the enormous size of possible combinations of brain regions. In the neuroimaging community, currently, there are two major streams of methodologies for functional connectivity study: ROI-based and clustering-based approaches. ROI-based approach is typically applied in applications that study limited number of brain regions. ROIs are either determined by manual placement of regions in the brain images or automatically determined by activation detection or other regional homogeneity analysis [4-6]. Clustering-based approaches are typically data-driven and they identify the brain networks that have more coherent fMRI time-series signals within each network [7, 8]. However, for high spatial resolution rsfMRI data, the computation time and memory consumption for volumetric clustering are increasing dramatically. 
In this paper, we propose an intuitive fiber-centered approach to study the functional connectivity between brain regions using high spatial resolution diffusion tensor imaging (DTI) and rsfMRI data. Our premise is that axonal fibers obtained from DTI data are the structural substrates of functional connectivity between brain regions, and thus provide a natural anatomical localization for inference of functional connectivity. Therefore, we measure the correlation between rsfMRI time series of two gray matter voxels that this fiber connects to define the functional coherence of the fiber and the functional connectivity between the voxels it connect. We applied the above methodology to study the functional coherences of all fibers in the entire brain and to study the functional connectivity between major brain regions, which are parcellated via an atlas-based warping algorithm [9].

\section{Method}

\subsection{Overview of the Method}

The proposed computational pipeline is summarized in Figure 1. Firstly, we coregister rsfMRI, DTI and T1 images into the same space using the FSL FLIRT tool (http://www.fmrib.ox.ac.uk/f $s l /)$. Then the whole brain is parcellated into regions by applying an atlas-based warping algorithms on the tissue map obtained from T1 image [9]. The anatomical labels are further mapped onto the DTI tissue map derived from [10]. After that, we group the tracked fibers from DTI data into clusters based on the brain

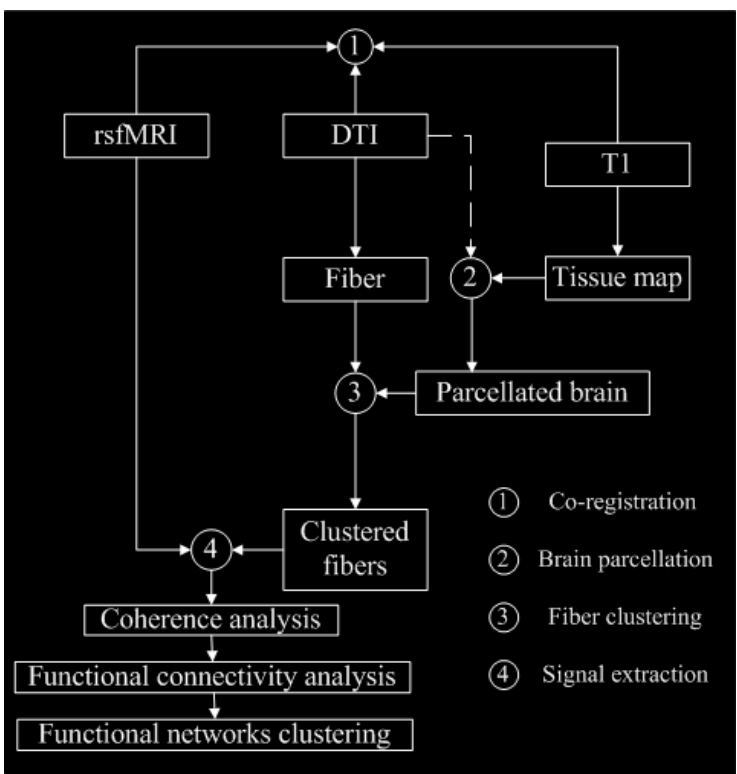

Fig. 1. The flowchart of the proposed computation pipeline for fiber-centered functional connectivity analysis. regions that they connect.

Finally, we extract the nearest gray matter voxels' rsfMRI signals for the two ends of each fiber, and perform functional coherence analysis, functional connectivity analysis and functional networks clustering based on these signals. This paper focuses on the last step.

\subsection{Data Acquisition and Preprocessing}

Eight volunteers were scanned using a 3T GE Signa MRI system. We acquired the resting state fMRI data with dimensionality $128 \times 128 \times 60 \times 100$, space resolution $2 m m \times 2 m m \times 2 m m$, TR $5 s$, TE $25 m s$, and flip angle 90 degrees. DTI data were acquired 
using the same spatial resolution as the fMRI data. Parameters were TR $15.5 s$ and TE 89.5ms, with 30 DWI gradient directions and 3 B0 volumes acquired.

Pre-processing of the rsfMRI data consists of brain skull removal, motion correction, spatial smoothing, temporal pre-whitening, slice time correction, global drift removal, and band pass filtering $(0.01 \mathrm{~Hz} 0.1 \mathrm{~Hz})$. The pre-processing of the DTI data consists of brain skull removal, motion correction, and eddy current correction. After the pre-processing, fiber tracking was performed using MEDINRIA (FA threshold: 0.2; minimum fiber length: 20; sampled by 4). Brain tissue segmentation was conducted on DTI data by our method in [10].

DTI space is used as the standard space from which to generate the GM segmentation and to report the brain network results on the cortical surface. Since fMRI and DTI sequences are both EPI sequences, their distortions tend to be similar and the misalignment between DTI and fMRI images is much less than that between T1 and fMRI images [11]. Co-registration between DTI and rsfMRI data is performed using the FSL FLIRT (http://www.fmrib.ox.ac.uk/fsl/).

\subsection{Fiber Projection}

There are a few reasons that cause the tracked fibers are not necessarily located on the cortex [12]. They include: 1) The FA values around the boundaries of gray matter and white matter are relatively low and the tractography procedure might stop before reaching the cortex. As a result, the tracked fibers will be within the cortical surface. 2) There is discrepancy in the brain tissue segmentation based on DTI data and the DTI tractography. In this case, the fiber could be either outside the cortex if the gray matter is over-segmented or inside the cortex if the gray matter is under-segmented [12]. Hence, in order to use the fiber connection information on the cortex, we project the fibers onto the cortical surface. If the end point of a fiber lies outside the cortex, we search along the fiber backwards the cortical surface. Otherwise, we extend the fiber towards the cortical surface. The searching process stops either when the fiber arrives at the surface or it exceeds a searching threshold. In very rare case when a fiber cannot reach the surface, we treat this fiber as an outlier and remove it. When a fiber are projected to the the surface, it has two HAMMER labels [9] of the surface patchs its ends pass through. Then, with the two labels, the fibers can be clustered into groups based on different combinations of start-end HAMMER label patterns, and these groups reflcet the structural connectivity between brain regions.

\subsection{Functional Coherence Analysis for Fibers}

As reported in the literature, the blood supply to the white matter is significantly lower than that of the cortex (less than one fourth) [13], and the BOLD contribution to the white matter is relatively low. So, the investigation of gray matter rsfMRI signals makes more sense. To ensure signals are extracted from the nearest gray matter voxel for each fiber end, after the co-registration of DTI and rsfMRI data, the tissue segmentation map of DTI image is used to guide the search for the nearest gray matter voxel. The searching method is similar to the one in Section 2.3, but this searching ends at the nearest voxel with gray matter label in the DTI-derived tissue segmentation image. Then, we extract the rsfMRI time series for two ends of each fiber, and define the functional coherence for each fiber as the absolute value of the Pearson's correlation coefficient of the two ends' rsfMRI signals. 


\subsection{Functional Connectivity between Brain Regions}

In many previous studies of functional connectivity analysis, the fMRI signals are typically averaged within a brain region first, e.g., in a ROI (region of interest), and then the correlations between the averaged signals are computed as the functional connectivity. However, if the fMRI time series in a brain region are not coherent, the averaging process will smooth out real activity patterns. In this paper, the functional connectivity between two brain regions is defined as the average functional coherence of the fibers connecting them. Therefore the analysis of functional connectivity between two regions is decomposed into the analysis of functional connectivity of two structurally connected voxels. In this way, we avoided the risk of averaging inhomogeneous fMRI signals in a brain region.

To have anatomical meaningful regions of the brain, we first apply the HAMMER algorithm to parcellate the brain tissue obtained in Section 2.2 into 84 ROIs including both major cortical and sub-cortical structures [9, 14]. Then, for each hemisphere, we regroup these regions into 7 categories: the frontal lobe, the temporal lobe, the occipital lobe, the parietal lobe, subcortical region, cingulate region and the lateral ventricle. Therefore, we have 14 larger brain regions in addition. Using the method in Section 2.4, functional coherences of all fibers in the entire brain are calculated. Then we average the functional coherence of fibers connecting two regions to measure the functional connectivity strength between the two regions. So we have a symmetrical functional connectivity matrix $C$ for all the 14 regions of the entire brain, and $c(i, j)$ measures the functional connectivity strength between region $i$ and region $j, 0<c(i, j)<1$.

\subsection{Cluster Brain Regions Using Affinity Propagation Algorithm}

The affinity propagation (AP) method [15] is a recently developed clustering method that can automatically determine the number of clusters in a population. It has been proved to have better performance than the traditional $K$-means clustering algorithm [15]. In general, The AP algorithm works by finding a set of exemplars in the data and assigning other data points to the exemplars. The AP algorithm approximates maximization of the sum of similarities to exemplars by recursively passing realvalued message along edge in a factor graph until a good set of exemplars and corresponding clusters emerges.

In this paper we use the AP algorithm to cluster the 84 regions into different functional networks, treating each region as a node in a network. There are two major steps in AP algorithm: similarity matrix computation and real-valued message passing, as summarized in following sections. Details on AP algorithm are referred to [15].

\subsubsection{Definition of Similarity Matrix}

The similarity matrix is obtained before the execution of the AP algorithm that simultaneously considers all data points as potential exemplars. We use the functional connectivity matrix as the similarity matrix. And the similarity between two regions is defined as $s(i, k)=-1 / c(i, k)$, and for each $i, s(i, i)$ is set to 0 . In this paper, we choose the maximum of the similarity multiplied by a constant value as self-similarity value $p$, and then we can obtain the expected clustering number by adjusting $p$ value. 


\subsubsection{Real-Valued Message Passing Algorithm}

Basically, there are two kinds of messages exchanged between data points, responsibility and availability, and each takes a different type of competition into account. The inputs are the pair-wise similarities and data point preferences.

In AP algorithm, the similarity $s(i, k)$ indicates how well the data point $k$ is suited to be the exemplar for data point $i$. The preference $s(i, i)$ is defined such that points with high values are more likely to be selected as exemplars. The number of identified exemplars is affected by both the values of the input preference and the messagingpassing procedure. The responsibility $r(i, k)$, sent from data point $i$ to a candidate exemplar point $k$, reflects the accumulated evidence of how well-suited point $k$ is to serve as the exemplar for point $i$, taking into account other potential exemplars for point $i$. The self-responsibility $r(k, k)$ reflects accumulated evidence that point $k$ is an exemplar based on its input preference tempered by how ill-suited it is to be assigned to another exemplar. The availability $a(i, k)$, sent from candidate exemplar point $k$ to point $i$, indicates the accumulated evidence of how appropriate it would be for point $i$ to choose point $k$ as its exemplar, taking into account the support from other points that point $k$ should be an exemplar. The availability $a(k, k)$, reflecting accumulated evidence that point $k$ is an exemplar, is based on the positive responsibilities sent to candidate exemplar $k$ from other points.

The update of responsibility lets all the candidate exemplars compete for ownership of a data point, whereas the update of availability collects evidence from data point as to whether each candidate exemplar would be a good exemplar. The messages of availability and responsibility are updated recursively. After convergence, availabilities and responsibilities are combined to identify exemplars. For point $i$, the value of $k$ that maximizes $a(i, k)+r(i, k)$ either identifies point $i$ as an exemplar if $k=i$, or identify data point that is the exemplar for point $i$. More details are referred to [15].

\section{Results}

\subsection{Distribution of Fibers' Functional Coherences}

We calculated the functional coherence of each fiber in the entire brain using the method mentioned in Section 2.4, and the statistic was done for all 8 subjects. We can conclude from Figure 2 that the functional coherence strength has a consistent probability distribution across subjects.

The functional coherence is also color coded for each fiber in the whole brain, as shown in

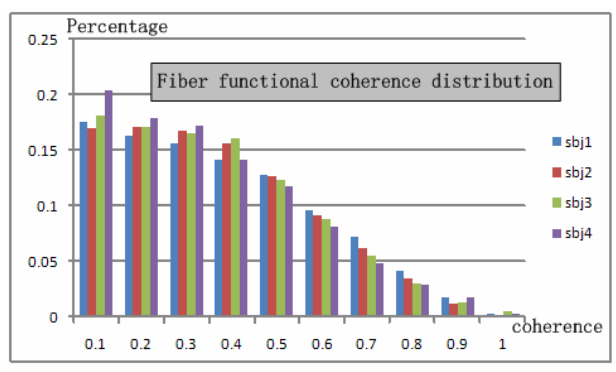

Fig. 2. Distributions of the fiber functional coherences of 4 randomly selected subjects

Figure 3. The color of each fiber stands for the strength of functional coherence, and 
the color bar is on the right. Figure 3 shows that the fiber functional coherence strength is correlated to the regions it connects. For instance, some groups of fibers connecting the temporal lobe have relatively lower functional coherences, while the inter-hemisphere fibers connecting the occipital lobes have relatively higher functional coherences. And this pattern is consistent across different brains, as shown in Figure 3 for 3 randomly selected subjects. This result demonstrates that the region-specific distribution of fibers' functional coherences is not random; it

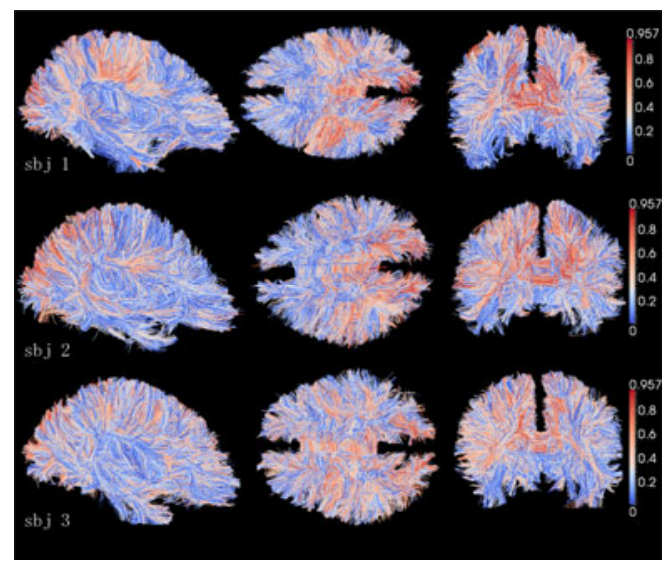

Fig. 3. Distributions of fibers' functional coherences over entire brain for 3 randomly selected subjects rather reflects brain's consistent functional architecture.

\subsection{Functional Connectivity Analysis}

Based on the 14 regions described in section 2.5 , we study the functional connectivity between these regions by constructing a connectivity matrix. We arrange the 14 regions in the order shown in Table.1. The distribution of the 14 regions is exactly symmetrical along the $\mathrm{Y}$ axis in Figure 4(a), which will help to view connectivity patterns.

The element $(x, y)$ of the connectivity matrix is defined by the averaged functional coherence of all fibers connecting the region $x$ and region $y$. Figure 4(a) shows the connectivity matrices for 4 randomly selected subjects. As we can see from the figure, 1) The connectivity matrix is relatively symmetrical along the dash dot line $R ; 2)$ The first and third quadrant is symmetrical along $R$, which means the functional connectivity between two hemispheres is relatively spatially symmetrical; 3) The second and fourth quadrant looks similar, demonstrating that the functional networks in each hemisphere are similar; 4) The connectivity pattern looks similar across different brains, but also with Table 1. Region index

\begin{tabular}{|l|l|l|}
\hline \multirow{2}{*}{ Region name } & \multicolumn{2}{|l|}{ Region ID } \\
\cline { 2 - 3 } & Left & Right \\
\hline Frontal lobe & 1 & 14 \\
\hline Temporal lobe & 2 & 13 \\
\hline Occipital lobe & 3 & 12 \\
\hline Parietal lobe & 4 & 11 \\
\hline Subcortical region & 5 & 10 \\
\hline Cingulate region & 6 & 9 \\
\hline Lateral ventricle & 7 & 8 \\
\hline
\end{tabular}
considerable variation.

Similarly, based on the brain parcellation in step 2 (Figure 1), we performed functional connectivity analysis for 84 brain regions. The 84 regions are also arranged in a symmetrical order. In other words, the 14 regions are decomposed into smaller parts without changing the Region ID, and the smaller parts are also arranged to be symmetrical along the $\mathrm{Y}$ axis. The connectivity matrices are visualized in Figure 4(b). The connectivity matrices for the 84 regions further strongly support the conclusions drawn from 14 regions analysis above. However, it reflects more discrepancy between 
two hemispheres, which is in consistency with the asymmetric brain. It is apparent that the connectivity pattern across subjects is quite reproducible. Also, the comparison of 14 and 84 regions analysis supports the idea that more segregated brain regions may have more functional differentiation.
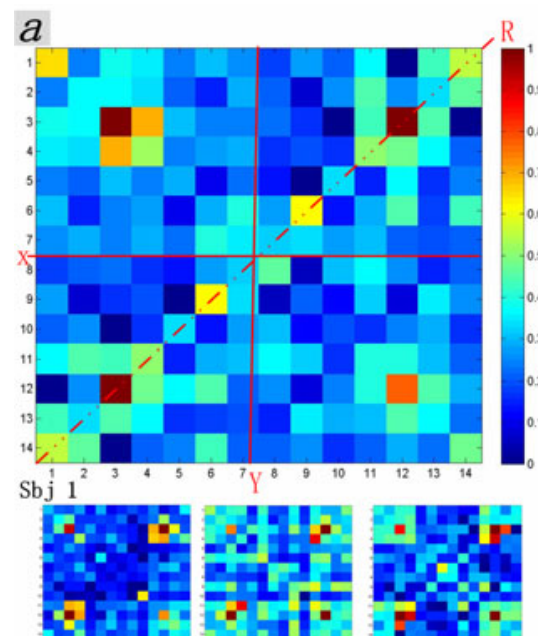

Sbj 2

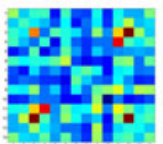

Sbj 3

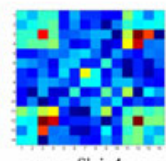

Sbj 4

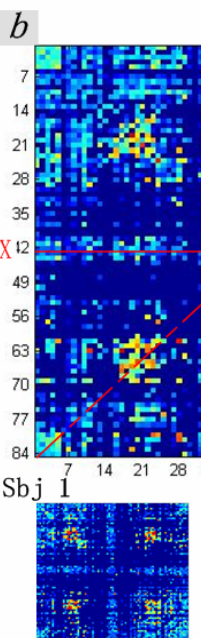

$\operatorname{Sbj} 2$

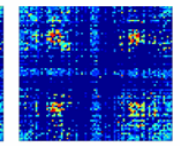

$\mathrm{Sbj} 3$

$\mathrm{R}$

Fig. 4. (a) Functional connectivity matrices of 14 regions for 4 randomly selected subjects. (b) Functional connectivity matrices of 84 regions for 4 randomly selected subjects.

\subsection{Region Clustering Using AP Algorithm}

As we discussed in the previous section, the functional connectivity matrices for 84 regions show a stable pattern across subjects, which makes the clustering via AP algorithm feasible. We adjust the self-similarity value $p$ in AP algorithm to obtain a stable clustering for each subject, and the cluster number for each case ranges from 20 to 24 . The clustering result is mapped to cortical surface, and for each subject each color represents a sub-network, as shown in Figure 5 for 4 randomly selected subjects. Note that the colors are just randomly selected to show sub-network

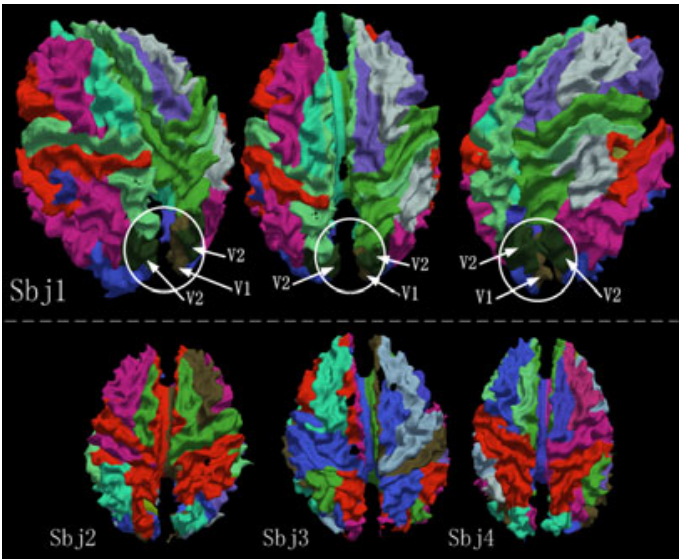

Fig. 5. Clustering of the 84 regions into subnetworks for randomly selected 4 regions

boundaries for each subject. They may vary across subjects for the same functional sub-network. The clustering result looks reasonable by visual inspection. As we can see in the top of Figure 5, the visual cortical regions of two hemispheres are clustered 
into meaningful regions, e.g., the V1 and V2 regions are clearly separated. This cluster is relatively consistent across subjects. We can see that the distributions of some subnetworks are symmetrical in two hemispheres. Some sub-networks contain regions across hemispheres. These clustering results support the view that the anatomical structural network is the basis of functional networks.

\section{Discussion and Conclusion}

In this paper, we present a novel fiber-centered approach for functional connectivity analysis in the entire brain. The advantages of this methodology include its intuitiveness and anatomical meaning. The analysis of functional connectivity between two brain regions is converted into the analysis of functional coherences of the fibers connecting these two regions. Our analysis on normal brains has shown meaningful results. In the future, we will cluster fibers using the functional coherences and their spatial distribution features. With the clustered fibers, we can potentially parcellate the white matter and gray matter into functionally homogenous regions automatically.

\section{References}

1. Fox, M.D., Raichle, M.E.: Spontaneous fluctuations in brain activity observed with functional magnetic resonance imaging. Nat. Rev. Neurosci. 8(9), 700-711 (2007)

2. Damoiseaux, J.S., et al.: Consistent resting-state networks across healthy subjects. PNAS, 13848-13853 (2006)

3. Honey, C.J., et al.: Predicting human resting-state functional connectivity from structural connectivity. PNAS 106(6), 2035-2040 (2009)

4. Biswal, B., et al.: Functional connectivity in the motor cortex of resting human brain using echo-planar MRI. Magn. Reson. Med. 34(4), 537-541 (1995)

5. Cordes, D., et al.: Mapping functionally related regions of brain with functional connectivity MR imaging. AJNR Am. J. Neuroradiol. 21(9), 1636-1644 (2000)

6. Biswal, B., et al.: Simultaneous assessment of flow and BOLD signals in resting-state functional connectivity maps. NMR Biomed. 10(4-5), 165-170 (1997)

7. De Luca, M., et al.: fMRI resting state networks define distinct modes of long-distance interactions in the human brain. Neuroimage 29(4), 1359-1367 (2006)

8. van den Heuvel, M., et al.: Normalized cut group clustering of resting-state FMRI data. PLoS One, 3(4), e2001 (2008)

9. Shen, D., et al.: HAMMER: hierarchical attribute matching mechanism for elastic registration. IEEE Trans. Med. Imaging 21(11), 1421-1439 (2002)

10. Liu, T., et al.: Brain Tissue Segmentation Based on DTI Data. NeuroImage 38(1), 114-123 (2007)

11. Li, K., et al.: Cortical surface based identification of brain networks using high spatial resolution resting state FMRI data. In: IEEE International Symposium on Biomedical Imaging (ISBI), Rotterdam, pp. 657-659 (2010)

12. Zhang, D., et al.: Automatic cortical surface parcellation based on fiber density information. In: IEEE International Symposium on Biomedical Imaging (ISBI), Rotterdam, pp. $1133-1136(2010)$

13. Mezer, A., et al.: Cluster analysis of resting-state fMRI time series. NeuroImage 45, 11171125 (2009)

14. Liu, T., et al.: Deformable Registration of Cortical Structures via Hybrid Volumetric and Surface Warping. NeuroImage 22(4), 1790-1801 (2004)

15. Frey, B.J., Dueck, D.: Clustering by Passing Messages between Data Points. Science 315, 972-976 (2007) 